PROCEEDINGS OF THE

AMERICAN MATHEMATICAL SOCIETY

Volume 128, Number 1, Pages 161-171

$\mathrm{S}$ 0002-9939(99)05110-2

Article electronically published on June 24, 1999

\title{
BOUNDS ON EMBEDDED SINGULAR SPECTRUM FOR ONE-DIMENSIONAL SCHRÖDINGER OPERATORS
}

\author{
CHRISTIAN REMLING
}

(Communicated by Christopher D. Sogge)

\begin{abstract}
We show that the solutions of the one-dimensional Schrödinger equation $-y^{\prime \prime}+V y=E y$ with potential $V(x)=O\left(x^{-\alpha}\right)$ satisfy the WKB asymptotic formulae off a set of energies $E$ of Hausdorff dimension $\leq 2(1-\alpha)$. This result gives restrictions on the structure of possible embedded singular spectrum. The proof relies on new norm estimates for the integral transform associated with the WKB method.
\end{abstract}

\section{INTRODUCTION}

This paper is, in a sense, a sequel to (the first part of) [12]. We are interested in one-dimensional Schrödinger equations,

$$
-y^{\prime \prime}(x)+V(x) y(x)=E y(x),
$$

with potentials $V$ satisfying

$$
|V(x)| \leq \frac{C}{(1+x)^{\alpha}}
$$

Eq. (1) describes the motion of a quantum mechanical particle, subject to an external field $V$. The physics of this system depends directly on the spectral properties of the corresponding operators (consult [11] for further background information); here, we'll treat explicitly only the half-line problem. So, we'll study the spectra of the self-adjoint operators

$$
H_{\beta}=-\frac{d^{2}}{d x^{2}}+V(x), \quad \beta \in[0, \pi),
$$

on $L_{2}(0, \infty)$ with boundary conditions $y(0) \cos \beta+y^{\prime}(0) \sin \beta=0$ (see, e.g., [19] for the general theory).

If $V$ is asymptotically small in a suitable sense, then, from physical considerations, one expects that $H_{\beta}$ has purely absolutely continuous spectrum on $(0, \infty)$. In fact, if $V$ tends to zero and doesn't oscillate too wildly, then one can use the so-called WKB methods to solve (1) asymptotically (cf. [5]). One gets solutions of the form

$$
\left(\begin{array}{c}
y(x, E) \\
y^{\prime}(x, E)
\end{array}\right)=\left(\begin{array}{c}
1 \\
i \sqrt{E}
\end{array}\right) e^{i \omega(x, E) / 2}+o(1) \quad(x \rightarrow \infty) .
$$

Received by the editors March 10, 1998.

1991 Mathematics Subject Classification. Primary 34L40, 81Q10.

(C)1999 American Mathematical Society 
The WKB phase $\omega$ will be defined below; it satisfies $\omega(x, E) \sim 2 \sqrt{E} x$. Since these solutions are, in particular, bounded, we can indeed deduce absence of singular spectrum [16].

Clearly, assumption (2) alone does not prevent $V$ from oscillating and hence does not ensure the applicability of the WKB methods. Indeed, classical [18] and more recent $[10,15]$ counterexamples show that there can be singular spectrum on $(0, \infty)$ if $\alpha \leq 1$. Moreover, if $\alpha \leq 1 / 2$, there are (random) examples with purely singular spectrum [4, 14]. However, as was recently proved by Christ-Kiselev [1] and the author [12] (see also the joint announcement [2]), this behavior is still exceptional if $\alpha>1 / 2$. More precisely, let

$$
S=\{E>0:(1) \text { does not have a solution } y \text { satisfying (3) }\} \text {. }
$$

Then the results of $[1,12]$, specialized to the situation where $V$ satisfies (2), say:

Theorem 1.1 ([1, 12]). Assume that (2) holds with $\alpha>1 / 2$. Then the exceptional set is of Lebesgue measure zero: $|S|=0$.

Again by [16], this result in particular implies that $\sigma_{a c}=[0, \infty)$. The main goal of this paper is to deepen our understanding of the behavior of the exceptional set $S$. We will prove the following strengthening of Theorem 1.1.

Theorem 1.2. Assume that (2) holds. Then the Hausdorff dimension of the exceptional set satisfies $\operatorname{dim} S \leq 2(1-\alpha)$.

I sincerely believe that this bound is optimal: It gives the correct values in the borderline cases $\alpha=1 / 2$ and $\alpha=1$, and it "interpolates" in a natural way. However, it's unclear to me how to construct examples substantiating this opinion.

There's an interesting difference between Theorems 1.1, 1.2: Theorem 1.1 remains true if one modifies $V$ by inserting arbitrary intervals with $V=0$ (this follows from [1, 12]). This is completely wrong for Theorem 1.2: If these intervals occur sufficiently frequently and are long enough, one always has $\operatorname{dim} S=1$ (see [13], where a stronger result is proved).

Of course, Theorem 1.2 is more than merely a statement on the solutions of (1). Since the singular part of the spectral measure (restricted to $(0, \infty)$ ) is supported by $S$ (note that this holds for all boundary conditions $\beta$ simultaneously), Theorem 1.2 immediately gives restrictions on the structure of possible embedded singular spectrum.

In fact, the picture is perhaps somewhat different from what could be expected naively. If $\alpha>1$, then the spectrum is purely absolutely continuous on $(0, \infty)$. For $\alpha \leq 1$, there may be singular spectrum as well, but the singular spectral measures that occur first (namely, for $\alpha=1$ ) are, so to speak, at the opposite end of the scale of measures: They are zero-dimensional! As $\alpha$ decreases further, the absolutely continuous spectrum persists as long as $\alpha>1 / 2$, while the "gap" of forbidden dimensions $(2(1-\alpha), 1)$ becomes smaller. It disappears at $\alpha=1 / 2$. It should also be kept in mind that it's not known if there really are potentials satisfying (2) with $\alpha>1 / 2$ with non-empty singular continuous spectrum.

Theorem 1.2 also has the following consequence:

Theorem 1.3. Assume that (2) holds. Then $H_{\beta}$ is purely absolutely continuous on $(0, \infty)$ for all $\beta \in[0, \pi)$ with the possible exception of a set of $\beta$ 's of Hausdorff dimension $\leq 2(1-\alpha)$. 
The plan of this paper is as follows. The proof of Theorem 1.3, given Theorem 1.2 , is based on the methods developed in [3]; it will be given in the last section. The proof of Theorem 1.2 rests on two pillars: First of all, we will establish a new norm estimate (= Theorem 3.1) on what I'd like to call the "WKB transform"

$$
(T f)(E) \equiv \int f(x) e^{i \omega(x, E)} d x .
$$

The importance of this operator was first recognized in [7]. Here, it will be vitally important to work in $L_{2}$-spaces with singular measures, in order to obtain the finer resolution needed to pass from Theorem 1.1 to Theorem 1.2. We will prove this norm estimate in Sections 2, 3, which are very much at the heart of this paper.

Second, we will need the machinery of [12]. In fact, Theorem 1.2 will follow from combining the methods of [12] with Theorem 3.1, plus some measure theory. This will be done in Section 4. To keep the length of this paper within reasonable bounds, we will have to be rather sketchy here; some further details will be provided in the Appendix.

\section{WKB tRANSFORMS OF D-DIMENSIONAL MEASURES}

Throughout this paper, $\mu$ will denote a finite Borel measure whose support is contained in some compact subset of $(0, \infty)$. Such a $\mu$ is said to be $D$-dimensional, with $D \in[0,1]$, if $\mu(I) \leq C|I|^{D}$ for all intervals $I \subset \mathbb{R}$.

It will be convenient to work with wavenumbers $k=\sqrt{E}$. Of course, the transformed set $\widetilde{S}=\left\{k>0: k^{2} \in S\right\}$ has the same Hausdorff dimension as $S$.

Given a potential $V$, we define the corresponding WKB phase $\omega=\omega_{V}$ by

$$
\omega(x, k)=2 k x-\frac{1}{k} \int_{0}^{x} V(t) d t
$$

As explained in the preceding section, this quantity plays a central role in the perturbation theory of the Schrödinger equation (1).

Our goal in this section is to prove the following result on WKB transforms of $D$-dimensional measures.

Theorem 2.1. Suppose that $\mu$ is D-dimensional with $D \in[0,1)$ and $|V(x)| \leq$ $C_{0}(1+x)^{-\alpha}$ for some $\alpha>1 / 2$. Then there is a constant $C=C\left(\mu, C_{0}, \alpha\right)$ so that

$$
\int_{0}^{L} d x\left|\int d \mu(k) f(k) e^{i \omega_{V}(x, k)}\right|^{2} \leq C L^{1-D} \int d \mu(k)|f(k)|^{2}
$$

for all $f \in L_{2}(\mathbb{R}, d \mu), L \geq 0$.

Remarks. 1. In the special case $V \equiv 0$, the Theorem gives estimates on the Fourier transform of $D$-dimensional measures. These results are due to Strichartz [17]; the proof is easier in this case. We will use some ideas from the proof of Strichartz's Theorem, as given in [9].

2. Since there will be so many different constants, we will, as usual, use the same letter $C$ for all of them. 
Proof. The assertion is obvious for $L<1$, so we may assume $L \geq 1$ throughout. Clearly,

$$
\begin{aligned}
& \int_{0}^{L} d x\left|\int d \mu(k) f(k) e^{i \omega(x, k)}\right|^{2} \\
& \quad \leq e \int_{-\infty}^{\infty} d x e^{-x^{2} / L^{2}}\left|\int d \mu(k) f(k) e^{i \omega(x, k)}\right|^{2} \\
& \quad=e \iint d \mu(k) d \mu\left(k^{\prime}\right) f(k) \overline{f\left(k^{\prime}\right)} \int_{-\infty}^{\infty} d x e^{-x^{2} / L^{2}} e^{2 i\left(k-k^{\prime}\right) x} e^{i\left(\frac{1}{k^{\prime}}-\frac{1}{k}\right) \int_{0}^{x} V(s) d s} .
\end{aligned}
$$

We integrate by parts in the last integral; in order to avoid convergence issues, we set $V=0$ on $(-\infty, 0)$.

$$
\begin{aligned}
& \int_{-\infty}^{\infty} d x e^{-x^{2} / L^{2}} e^{2 i\left(k-k^{\prime}\right) x} e^{i\left(\frac{1}{k^{\prime}}-\frac{1}{k}\right) \int_{0}^{x} V(s) d s}=\int_{-\infty}^{\infty} d x e^{-x^{2} / L^{2}} e^{2 i\left(k-k^{\prime}\right) x} \\
& \quad+i\left(\frac{1}{k^{\prime}}-\frac{1}{k}\right) \int_{-\infty}^{\infty} d x V(x) e^{i\left(\frac{1}{k^{\prime}}-\frac{1}{k}\right) \int_{0}^{x} V(s) d s} \int_{x}^{\infty} d t e^{-t^{2} / L^{2}} e^{2 i\left(k-k^{\prime}\right) t}
\end{aligned}
$$

In the first integral (6a), we've gotten rid of the $V$ in the exponent, and thus the corresponding term can be estimated as in the case of the Fourier transform. We repeat this argument here for completeness: First of all, the $x$-integral can be evaluated, and after inserting it into (5) we get

$$
\begin{aligned}
& C L \iint d \mu(k) d \mu\left(k^{\prime}\right) f(k) \overline{f\left(k^{\prime}\right)} e^{-\left(k-k^{\prime}\right)^{2} L^{2}} \\
& \quad \leq C L \int d \mu(k)|f(k)|^{2} \int d \mu\left(k^{\prime}\right) e^{-\left(k-k^{\prime}\right)^{2} L^{2}} .
\end{aligned}
$$

Now,

$$
\begin{aligned}
\int d \mu\left(k^{\prime}\right) e^{-\left(k-k^{\prime}\right)^{2} L^{2}} & =\sum_{n=0}^{\infty} \int_{n / L \leq\left|k-k^{\prime}\right|<(n+1) / L} d \mu\left(k^{\prime}\right) e^{-\left(k-k^{\prime}\right)^{2} L^{2}} \\
& \leq C L^{-D} \sum_{n=0}^{\infty} e^{-n^{2}}=C L^{-D}
\end{aligned}
$$

so we get indeed the desired bound on (5).

It remains to study the contribution coming from (6b). The basic strategy is as follows. We'll break up the double integral over $k, k^{\prime}$ into two parts, according to the size of $\left|k-k^{\prime}\right|$. Since the $t$-integral from (6b) is oscillatory, we expect that it decays as $\left|k-k^{\prime}\right|$ grows. Note that a similar effect made the above argument work. For small $\left|k-k^{\prime}\right|$, there are no such cancellations, but then the $k, k^{\prime}$ integration is only over a small region.

We start by integrating by parts in the $t$-integral:

$$
\begin{aligned}
\int_{x}^{\infty} d t e^{-t^{2} / L^{2}} e^{2 i\left(k-k^{\prime}\right) t}= & \frac{-1}{2 i\left(k-k^{\prime}\right)} e^{2 i\left(k-k^{\prime}\right) x} e^{-x^{2} / L^{2}} \\
& +\frac{1}{i\left(k-k^{\prime}\right) L^{2}} \int_{x}^{\infty} d t t e^{-t^{2} / L^{2}} e^{2 i\left(k-k^{\prime}\right) t}
\end{aligned}
$$


Let's first look at the contribution coming from (7a). So insert (7a) into (6b), and then plug the resulting term into (5). We get

$$
\begin{aligned}
C \iint d \mu(k) d \mu\left(k^{\prime}\right) \frac{f(k) \overline{f\left(k^{\prime}\right)}}{k k^{\prime}} & \int_{-\infty}^{\infty} d x V(x) e^{-x^{2} / L^{2}} e^{2 i\left(k-k^{\prime}\right) x} e^{i\left(\frac{1}{k^{\prime}}-\frac{1}{k}\right) \int_{0}^{x} V(s) d s} \\
= & C \int_{-\infty}^{\infty} d x V(x) e^{-x^{2} / L^{2}}\left|\int d \mu(k) \frac{f(k)}{k} e^{i \omega(x, k)}\right|^{2},
\end{aligned}
$$

and the absolute value of this expression can obviously be estimated by

$$
\begin{aligned}
C \int_{-\infty}^{\infty} & d x\left(1+x^{2}\right)^{-\alpha / 2} e^{-x^{2} / L^{2}}\left|\int d \mu(k) \frac{f(k)}{k} e^{i \omega(x, k)}\right|^{2} \\
= & C \iint d \mu(k) d \mu\left(k^{\prime}\right) \frac{f(k) \overline{f\left(k^{\prime}\right)}}{k k^{\prime}} \\
& \times \int_{-\infty}^{\infty} d x\left(1+x^{2}\right)^{-\alpha / 2} e^{-x^{2} / L^{2}} e^{2 i\left(k-k^{\prime}\right) x} e^{i\left(\frac{1}{k^{\prime}}-\frac{1}{k}\right) \int_{0}^{x} V(s) d s} .
\end{aligned}
$$

As announced above, we now break up this triple integral into two parts, according as $\left|k-k^{\prime}\right| \leq L^{\alpha / D-1}$ or $\left|k-k^{\prime}\right|>L^{\alpha / D-1}$. (Of course, we assume $D>0$ here; if $D=0$, the assertion is trivial.) Consider first the region $\left|k-k^{\prime}\right| \leq L^{\alpha / D-1}$ : We don't expect cancellations in the $x$-integral here, so we simply use

$$
\begin{aligned}
\mid \int_{-\infty}^{\infty} d x\left(1+x^{2}\right)^{-\alpha / 2} e^{-x^{2} / L^{2}} e^{2 i\left(k-k^{\prime}\right) x} & e^{i\left(\frac{1}{k^{\prime}}-\frac{1}{k}\right) \int_{0}^{x} V(s) d s} \mid \\
\leq & \int_{-\infty}^{\infty} d x\left(1+x^{2}\right)^{-\alpha / 2} e^{-x^{2} / L^{2}} \leq C L^{1-\alpha} .
\end{aligned}
$$

This yields the required bound on the corresponding part of (8):

$$
\begin{array}{rl}
C L^{1-\alpha} \iint_{\left|k-k^{\prime}\right| \leq L^{\alpha / D-1}} & d \mu(k) d \mu\left(k^{\prime}\right)\left|f(k) f\left(k^{\prime}\right)\right| \\
\leq C L^{1-\alpha} \int d \mu(k)|f(k)|^{2} \int_{\left|k-k^{\prime}\right| \leq L^{\alpha / D-1}} d \mu\left(k^{\prime}\right) \\
\leq C L^{1-\alpha+(\alpha / D-1) D} \int d \mu(k)|f(k)|^{2} \\
=C L^{1-D} \int d \mu(k)|f(k)|^{2} .
\end{array}
$$

To analyze the part of (8) corresponding to $\left|k-k^{\prime}\right|>L^{\alpha / D-1}$, we use once again integration by parts in the $x$-integral:

(9)

$$
\begin{gathered}
\int_{-\infty}^{\infty} d x\left(1+x^{2}\right)^{-\alpha / 2} e^{-x^{2} / L^{2}} e^{2 i\left(k-k^{\prime}\right) x} e^{i\left(\frac{1}{k^{\prime}}-\frac{1}{k}\right) \int_{0}^{x} V(s) d s} \\
=\int_{-\infty}^{\infty} d x\left(1+x^{2}\right)^{-\alpha / 2} e^{i\left(\frac{1}{k^{\prime}}-\frac{1}{k}\right) \int_{0}^{x} V(s) d s}\left[-\alpha x\left(1+x^{2}\right)^{-1}+i\left(\frac{1}{k^{\prime}}-\frac{1}{k}\right) V(x)\right] \\
\times \int_{x}^{\infty} d t e^{-t^{2} / L^{2}} e^{2 i\left(k-k^{\prime}\right) t} .
\end{gathered}
$$

Now integrate by parts in the $t$-integral (this is the usual method to exploit oscillations). We did this calculation already in (7a), (7b). It follows from these formulae 
that

$$
\left|\int_{x}^{\infty} d t e^{-t^{2} / L^{2}} e^{2 i\left(k-k^{\prime}\right) t}\right| \leq \frac{C}{\left|k-k^{\prime}\right|}
$$

and thus (9) implies that also

$$
\left|\int_{-\infty}^{\infty} d x\left(1+x^{2}\right)^{-\alpha / 2} e^{-x^{2} / L^{2}} e^{2 i\left(k-k^{\prime}\right) x} e^{i\left(\frac{1}{k^{\prime}}-\frac{1}{k}\right) \int_{0}^{x} V(s) d s}\right| \leq \frac{C}{\left|k-k^{\prime}\right|}
$$

Therefore, going back to (8), we find the bound

$$
\begin{aligned}
& C \iint_{\left|k-k^{\prime}\right|>L^{\alpha / D-1}} d \mu(k) d \mu\left(k^{\prime}\right) \frac{\left|f(k) f\left(k^{\prime}\right)\right|}{\left|k-k^{\prime}\right|} \\
& \quad \leq C \int d \mu(k)|f(k)|^{2} \int_{\left|k-k^{\prime}\right|>L^{\alpha / D-1}} \frac{d \mu\left(k^{\prime}\right)}{\left|k-k^{\prime}\right|} \\
& \quad \leq C L^{(1-D+\epsilon)(1-\alpha / D)} \int d \mu(k)|f(k)|^{2} \int_{\left|k-k^{\prime}\right|>L^{\alpha / D-1}} \frac{d \mu\left(k^{\prime}\right)}{\left|k-k^{\prime}\right|^{D-\epsilon}} .
\end{aligned}
$$

Now we need the following elementary fact:

Lemma 2.2. If $\mu$ is $D$-dimensional, then, for any $\epsilon \in(0, D)$,

$$
\sup _{x \in \mathbb{R}} \int \frac{d \mu(y)}{|x-y|^{D-\epsilon}}<\infty .
$$

Proof.

$$
\begin{aligned}
\int \frac{d \mu(y)}{|x-y|^{D-\epsilon}} & =\sum_{n=1}^{\infty} \int_{(n+1)^{-1 / \epsilon}<|x-y| \leq n^{-1 / \epsilon}} \frac{d \mu(y)}{|x-y|^{D-\epsilon}}+\int_{|x-y|>1} \frac{d \mu(y)}{|x-y|^{D-\epsilon}} \\
& \leq C \sum_{n=1}^{\infty}(n+1)^{(D-\epsilon) / \epsilon} n^{(-1-1 / \epsilon) D}+\mu(\mathbb{R}) \equiv C<\infty
\end{aligned}
$$

Hence, if $\epsilon>0$ is small enough, then equation (10) can indeed be estimated by $C L^{1-D}\|f\|_{L_{2}(\mathbb{R}, d \mu)}^{2}$. This concludes the analysis of the contributions coming from (7a).

We now move on to studying (7b). The same break-up as above will be used. If $\left|k-k^{\prime}\right| \leq L^{\alpha / D-1}$, note that

$$
|(7 \mathrm{~b})| \leq \frac{C}{\left|k-k^{\prime}\right| L^{2}} \int_{x}^{\infty} d t t e^{-t^{2} / L^{2}} \leq \frac{C}{\left|k-k^{\prime}\right|} e^{-x^{2} /\left(2 L^{2}\right)}
$$

hence again

$$
\left|\left(\frac{1}{k^{\prime}}-\frac{1}{k}\right) \int_{-\infty}^{\infty} d x V(x) e^{i\left(\frac{1}{k^{\prime}}-\frac{1}{k}\right) \int_{0}^{x} V(s) d s} \times(7 \mathrm{~b})\right| \leq C L^{1-\alpha},
$$

and proceed as above.

If $\left|k-k^{\prime}\right|>L^{\alpha / D-1}$, we integrate by parts one more time:

$$
(7 \mathrm{~b})=\frac{1}{2\left(k-k^{\prime}\right)^{2} L^{2}}\left(x e^{-x^{2} / L^{2}} e^{2 i\left(k-k^{\prime}\right) x}+\int_{x}^{\infty} d t\left(1-\frac{2 t^{2}}{L^{2}}\right) e^{-t^{2} / L^{2}} e^{2 i\left(k-k^{\prime}\right) t}\right) .
$$

It's easy to see that this gives an estimate on the corresponding contribution to (6b) of the form $C /\left(\left|k-k^{\prime}\right| L^{\alpha}\right)$. So we can again conclude the argument as above (in fact, we even have an additional small factor $L^{-\alpha}$ ). 


\section{Norm EStimates}

The result of the previous section enables us to show

Theorem 3.1. Suppose that $\mu$ is D-dimensional $(D \in[0,1])$ and $|V(x)| \leq$ $C_{0}(1+x)^{-\alpha}$ with $\alpha>1 / 2$. Then there is a constant $C$ so that

$$
\int d \mu(k)\left|\int_{c}^{d} d x f(x) e^{i \omega_{V}(x, k)}\right|^{2} \leq C(d-c)^{1-D} \int_{c}^{d} d x|f(x)|^{2}
$$

for all $f \in L_{2}(c, d), 0 \leq c<d<\infty$.

Remark. For $D=1$, this result is due to Kiselev [7]. It was an important ingredient to studying stability of a spectrum [1, 12]. Note also that if $D=1$ and $V \equiv 0$, then, of course, the assertion is an immediate consequence of Plancherel's formula.

Proof. Let

$$
g(k)=\int_{c}^{d} d x f(x) e^{i \omega_{V}(x, k)},
$$

and $\phi(k)=\arg (g(k))$. Then (using the notation $\left.h_{c}(x) \equiv h(x+c)\right)$

$$
\begin{aligned}
\int d \mu & (k)|g(k)|^{2}=\int d \mu(k)|g(k)| e^{-i \phi(k)} \int_{c}^{d} d x f(x) e^{i \omega_{V}(x, k)} \\
& =\int_{c}^{d} d x f(x) \int d \mu(k)|g(k)| e^{-i \phi(k)} e^{i \omega_{V}(x, k)} \\
& =\int_{0}^{d-c} d x f_{c}(x) \int d \mu(k)|g(k)| e^{i\left(\omega_{V}(c, k)-\phi(k)\right)} e^{i \omega_{V_{c}}(x, k)} \\
\leq & \|f\|_{L_{2}(c, d)}\left(\int_{0}^{d-c} d x\left|\int d \mu(k)\right| g(k)\left|e^{i\left(\omega_{V}(c, k)-\phi(k)\right)} e^{i \omega_{V_{c}}(x, k)}\right|^{2}\right)^{1 / 2} .
\end{aligned}
$$

Now Theorem 2.1 yields

$$
\|g\|_{L_{2}(\mathbb{R}, d \mu)}^{2} \leq C\|f\|_{L_{2}(c, d)}(d-c)^{(1-D) / 2}\|g\|_{L_{2}(\mathbb{R}, d \mu)} .
$$

Here, it's important that the constant $C$ from Theorem 2.1 depends only on the bound on $V$ (via $C_{0}, \alpha$ ) and not on the particular form of $V$ itself. Thus, since $V_{c}$ also satisfies (2) if $V$ does, the constant in (11) is independent of $c$.

Now divide (11) by $\|g\|_{L_{2}(\mathbb{R}, d \mu)}$ and then take squares.

\section{Proof of Theorem 1.2}

We now want to use the machinery of [12], with Theorem 3.1 as important additional input. Unfortunately, these considerations are rather technical and involved. Therefore, we will only state here what one can prove along these lines. A bird's eye view on the whole argument will be given in the Appendix; for details, the reader should consult [12].

Suppose $V$ satisfies (2) and $\mu$ is a $D$-dimensional measure with $D>2(1-\alpha)$ ( $\alpha$ is from (2), of course). Then $\mu(S)=0$, where $S$ is the exceptional set defined in $(4)$.

Accepting this, we can now prove Theorem 1.2 as follows. Assume that, contrary to the claim, $\operatorname{dim} S>2(1-\alpha)$. Then, by definition of the Hausdorff dimension, there exists $D>2(1-\alpha)$ so that $h^{D}(S)=\infty\left(h^{D}\right.$ denotes the $D$-dimensional Hausdorff 
measure). It's easy to see that $S$ is a Borel set. Hence, by [6, Theorem 5.6], we can find a compact subset $S^{\prime} \subset S$, such that $0<h^{D}\left(S^{\prime}\right)<\infty$ and $h^{D}\left(I \cap S^{\prime}\right) \leq C|I|^{D}$ for all intervals $I$. Now we can take as measure $\mu$ the restriction of $h^{D}$ to $S^{\prime}$ (i.e., $\left.\mu(F)=h^{D}\left(F \cap S^{\prime}\right)\right)$. Then the result from the preceding paragraph says that $\mu(S)=h^{D}\left(S^{\prime}\right)=0$, a contradiction.

\section{Proof of Theorem 1.3}

By general principles, possible singular spectral measure on $(0, \infty)$ is supported on $S$ for all $\beta \in[0, \pi)$. Hence it suffices to prove the following general result, which is closely related to [3, Theorem 5.2].

Theorem 5.1. For a Borel set $T \subset \mathbb{R}$, let

$$
A=\left\{\beta \in[0, \pi): \rho_{\beta}(T)>0\right\}
$$

(where $\rho_{\beta}$ is the spectral measure of $H_{\beta}$ ). Then $\operatorname{dim} A \leq \operatorname{dim} T$.

Proof. Let

$$
B=\{-\cot \beta: \beta \in A, \beta \neq 0\} .
$$

Clearly, $\operatorname{dim} A=\operatorname{dim} B$, so we may as well work with $B$. Now, assume that $h^{D}(B)=\infty$ for some $D<1$. Then, invoking again the argument based on $[6$, Theorem 5.6], we see that there's a $D$-dimensional measure $\mu$, supported by $B$ and with $0<\mu(B)<\infty$.

Define another measure $\nu$ by

$$
\nu(F)=\int \rho_{-\cot ^{-1} x}(F) \frac{d \mu(x)}{1+x^{2}} .
$$

Denote the Borel transform of a measure $\eta$ by $M_{\eta}$ :

$$
M_{\eta}(z) \equiv \int \frac{d \eta(t)}{t-z}
$$

Then [3, Lemma 5.3] (slightly modified to apply to the case at hand) says that

$$
M_{\nu}(z)=M_{\mu}\left(m_{0}(z)\right),
$$

where $m_{0}$ is the usual Titchmarsh-Weyl $m$-function of $H_{0}$.

Since $\mu$ is $D$-dimensional, we have that

$$
\left|M_{\mu}(E+i \epsilon)\right| \leq C \epsilon^{D-1} .
$$

This is well-known and can be shown by an argument similar to the proof of Lemma 2.2. Combining (12) and (13) yields

$$
\epsilon^{1-D}\left|M_{\nu}(E+i \epsilon)\right| \leq C\left(\frac{\epsilon}{\operatorname{Im} m_{0}(E+i \epsilon)}\right)^{1-D} .
$$

Since

$$
\frac{\operatorname{Im} m_{0}(E+i \epsilon)}{\epsilon}=\int \frac{d \rho_{0}(t)}{(t-E)^{2}+\epsilon^{2}},
$$

we see that in particular

$$
\limsup _{\epsilon \rightarrow 0+} \epsilon^{1-D}\left|M_{\nu}(E+i \epsilon)\right|<\infty
$$


for all $E \in \mathbb{R}$. This implies (cf. [3, Theorem 3.1])

$$
\left(D_{D} \nu\right)(E) \equiv \limsup _{\epsilon \rightarrow 0+} \frac{\nu(E-\epsilon, E+\epsilon)}{(2 \epsilon)^{D}}<\infty
$$

for all $E$. Hence $\nu(F)=0$ for every set $F$ with $\operatorname{dim} F<D$. But obviously $\nu(T)>0$; thus $\operatorname{dim} T \geq D$. The only assumption on $D$ was $h^{D}(B)=\infty$, and this holds for any $D<\operatorname{dim} B$, so we conclude that indeed $\operatorname{dim} T \geq \operatorname{dim} B$.

\section{Appendix A. The method of [12]}

First of all, we rewrite (1) using (modified) Prüfer variables. So, if $y(x, k)$ is a solution of (1) with $E=k^{2}$ (fixed via initial values at $x=0$ ), write $y=R \sin \psi / 2, y^{\prime}=$ $k R \cos \psi / 2$. The equations for $R$ and $\theta \equiv \psi-\omega$ are, in integrated form,

$$
\begin{aligned}
2 k(\ln R(y, k)-\ln R(x, k)) & =\int_{x}^{y} V(t) \sin \psi(t, k) d t, \\
k(\theta(y, k)-\theta(x, k)) & =\int_{x}^{y} V(t) \cos \psi(t, k) d t .
\end{aligned}
$$

We want to show that $R$ and $\theta$ tend to limits as $x \rightarrow \infty$, for $\mu$-a.e. $k$. Let $x_{n} \geq 0$ be an increasing sequence that tends to infinity, and write $V_{n}$ for the restriction of $V$ to the interval $\left(x_{n-1}, x_{n}\right)$. Eqs. (14), (15) suggest that we study

$$
\sum_{n=1}^{\infty} \int V_{n}(x) e^{i \psi(x, k)} d x=\sum_{n=1}^{\infty} \int V_{n}(x) e^{i \omega(x, k)} e^{i \theta(x, k)} d x .
$$

In fact, it turns out that for appropriately chosen $x_{n}$ 's, these sums are (absolutely) convergent for almost every $k$ with respect to $\mu$ if $\mu$ is as in Sect. 4 .

To prove this, one uses integration by parts, integrating $V_{n} e^{i \omega}$ and differentiating $e^{i \theta}$. This gives

$$
\begin{aligned}
& \sum_{n=1}^{\infty}\left(e^{i \theta\left(x_{n}, k\right)} \int V_{n}(x) e^{i \omega(x, k)} d x\right. \\
& \left.\quad-\frac{i}{k} \int d x V_{n}(x) \cos (\omega(x, k)+\theta(x, k)) e^{i \theta(x, k)} \int_{x_{n-1}}^{x} d t V_{n}(t) e^{i \omega(t, k)}\right) .
\end{aligned}
$$

Now Theorem 3.1 together with the Cauchy-Schwarz inequality (in $L_{2}(d \mu)$ ) show that the $\mu$-integral of the first term in the sum can be estimated by

$$
\int d \mu(k)\left|\int V_{n}(x) e^{i \omega(x, k)} d x\right| \leq C\left(x_{n}-x_{n-1}\right)^{(1-D) / 2}\left\|V_{n}\right\|_{2} .
$$

In particular, if the $x_{n}$ 's are chosen in such a way that this bound is summable (and we will do so shortly), then also

$$
\sum_{n=1}^{\infty}\left|\int V_{n}(x) e^{i \omega(x, k)} d x\right|<\infty
$$

for $\mu$-almost every $k$.

As for the remaining terms, it's possible to set up an iteration scheme also based on these two devices: integration by parts and the control over integrals with respect to $d \mu$ provided by Theorem 3.1. More specifically, one starts with the intervals $\left(x_{n-1}, x_{n}\right)$, and at each step, every interval is subdivided into smaller subintervals. The point is that the double integrals are over smaller and smaller 
regions (modulo contributions that are summable $\mu$-almost everywhere). Finally, an elementary estimate shows that these terms are also summable almost everywhere (after a sufficiently large number of steps).

In the case at hand, this procedure works if we take (sticking to the notation of [12]) $x_{n}=n^{a}$ with $a>\frac{D}{D-2(1-\alpha)}$ (here, we need the basic assumption $D>2(1-\alpha)$ to ensure that the denominator is positive) and $N_{n}=\left[n^{b}\right]$ with $0<b<1-D+$ $a(D-2(1-\alpha))$ and subdivide into $N_{n}$ subintervals of equal length at each step. This is proved by a careful step-by-step check of the arguments of [12, Sect. 2].

In this way, one establishes existence of the $\operatorname{limits}_{\lim _{n \rightarrow \infty}} R\left(x_{n}\right), \theta\left(x_{n}\right)$. The extension to sequences tending to infinity arbitrarily is based on a norm estimate for the maximal function

$$
M_{n}(k) \equiv \max _{x_{n-1} \leq \xi \leq x_{n}}\left|\int_{x_{n-1}}^{\xi} V(x) e^{i \omega(x, k)} d x\right|:
$$

Namely, we have that for $q>2,1 / p+1 / q=1$,

$$
\left\|M_{n}\right\|_{L_{q}(\mathbb{R}, d \mu)} \leq C_{q}\left(x_{n}-x_{n-1}\right)^{(1-D) / q}\left\|V_{n}\right\|_{p} .
$$

This is proved by interpolation: Since, trivially, the WKB transform is bounded as a map from $L_{1}$ to $L_{\infty}$ with norm 1 , the Riesz-Thorin Theorem [20, Chapter XII, 1.11] shows that the claimed estimate holds for the WKB transform itself. Then the results of [8] and [1, Sect. 3] guarantee that it holds for the maximal function as well.

Now, if we take $q$ sufficiently close to 2 , we can argue as in [12, Sect. 3] to prove that also

$$
\lim _{n \rightarrow \infty} \max _{x_{n-1} \leq \xi \leq x_{n}}\left|\int_{x_{n-1}}^{\xi} V(x) e^{i \psi(x, k)} d x\right|=0
$$

for $\mu$-almost every $k$.

\section{REFERENCES}

[1] M. Christ and A. Kiselev, Absolutely continuous spectrum for one-dimensional Schrödinger operators with slowly decaying potentials: Some optimal results, to appear in J. Amer. Math. Soc. CMP 98:12

[2] M. Christ, A. Kiselev, and C. Remling, The absolutely continuous spectrum of onedimensional Schrödinger operators with decaying potentials, Math. Research Letters 4 (1997), 719-723. CMP 98:05

[3] R. del Rio, S. Jitomirskaya, Y. Last, and B. Simon, Operators with singular continuous spectrum, IV. Hausdorff dimensions, rank one perturbations, and localization, J. d'Analyse Math. 69 (1996), 153-200. MR 97m:47002

[4] F. Delyon, B. Simon, and B. Souillard, From power pure point to continuous spectrum in disordered systems, Ann. Inst. H. Poincaré 42 (1985), 283-309. MR 87d:35098

[5] M.S.P. Eastham, The Asymptotic Solution of Linear Differential Systems, London Math. Soc. Monographs New Series 4, Clarendon Press, Oxford, 1989. MR 91d:34001

[6] K.J. Falconer, The Geometry of Fractal Sets, Cambridge University Press, Cambridge, 1985. MR 88d:28001

[7] A. Kiselev, Preservation of the absolutely continuous spectrum of Schrödinger equations under perturbations by slowly decreasing potentials and a.e. convergence of integral operators, Duke Math. J. 94 (1998), 619-646. CMP 98:17

[8] A. Kiselev, Interpolation theorem related to a.e. convergence of integral operators, to appear in Proc. Amer. Math. Soc.

[9] Y. Last, Quantum dynamics and decompositions of singular continuous spectra, J. Funct. Anal. 142 (1996), 406-445. MR 97k:81044 
[10] S.N. Naboko, Dense point spectra of Schrödinger and Dirac operators, Theor. and Math. Phys. 68 (1986), 646-653. MR 88h:81029

[11] M. Reed and B. Simon, Methods of Modern Mathematical Physics, III. Scattering Theory, Academic Press, London-San Diego, 1979. MR 80m:81085

[12] C. Remling, The absolutely continuous spectrum of one-dimensional Schrödinger operators with decaying potentials, Commun. Math. Phys. 193 (1998), 151-170. CMP 98:11

[13] C. Remling, Embedded singular continuous spectrum for one-dimensional Schrödinger operators, to appear in Trans. Amer. Math. Soc.

[14] B. Simon, Some Jacobi matrices with decaying potentials and dense point spectrum, Commun. Math. Phys. 87 (1982), 253-258. MR 85d:47033

[15] B. Simon, Some Schrödinger operators with dense point spectrum, Proc. Amer. Math. Soc. 125 (1997), 203-208. MR 97c:34179

[16] G. Stolz, Bounded solutions and absolute continuity of Sturm-Liouville operators, J. Math. Anal. Appl. 169 (1992), 210-228. MR 93f:34141

[17] R. Strichartz, Fourier asymptotics of fractal measures, J. Funct. Anal. 89 (1990), 154-187. MR 91m:42015

[18] J. von Neumann and E. Wigner, Über merkwürdige diskrete Eigenwerte, Z. Phys. 30 (1929), 465-467.

[19] J. Weidmann, Spectral Theory of Ordinary Differential Operators, Springer Lect. Notes 1258, Springer-Verlag, Berlin, 1987. MR 89b:47070

[20] A. Zygmund, Trigonometric Series, vol. I, II, Cambridge University Press, Cambridge, 1959. MR 21:6498

Universität Osnabrück, Fachbereich Mathematik/Informatik, 49069 OsnabrüCk, Germany

E-mail address: cremling@mathematik.uni-osnabrueck.de 